\title{
COMPOSIÇÃO DE LIPÍDIOS E ÁCIDOS GRAXOS DE Spirulina sp. (LEB18) PRODUZIDA EM ÁGUA RESIDUAL DA AQUICULTURA.
}

Isabella Borges dos Anjos da Silva1; Lucas Guimarães Cardoso2; Jessica Hartwig Duarte $^{3}$; Jorge Alberto Vieira Costa3; Denilson de Jesus Assis"; Janice Izabel Druzian $^{5}$; Fabio Alexandre Chinalia ${ }^{2}$; Karina Lizzeth Pedraza Galván ${ }^{5}$

${ }^{1}$ Faculdade de Farmácia, Universidade Federal da Bahia; Salvador/BA; isaanjos99@hotmail.com

IInstituto de Ciências da Saúde, Departamento de Biotecnologia, Universidade Federal da Bahia; Salvador/BA

${ }^{3}$ Laboratório de Engenharia Bioquímica, Faculdade de Química e Engenharia de Alimentos, Universidade Federal do Rio Grande; Rio Grande/RS

${ }^{4}$ Escola de Arquitetura, Engenharia e Tecnologia da Informação, Universidade de Salvador; Salvador/BA

${ }^{5}$ Programa de Graduação em Ciências dos Alimentos, Faculdade de Farmácia, Universidade Federal da Bahia; Salvador/BA

Resumo: O objetivo foi avaliar a composição de lipídios e ácidos graxos de Spirulina sp. (LEB 18) cultivada em água residual de aquicultura. Os cultivos foram realizados em fotobiorreatores (1L) com $100 \%$ de água residual de aquicultura suplementada com T-25, T-50, T-75. Os maiores teores de ácidos graxos poliinsaturados $(38,20 \%$ e $40,66 \%$ ) e C18:3n6 (38,20\% e 33,50\%) foram encontrados em $25 \%$ e $50 \%$, respectivamente. Assim, os tratamentos com 25\% e 50\% representam uma alternativa eficiente, barata e sustentável para o setor de aquicultura, reduzindo os impactos das descargas de efluentes, produzindo biomassa de baixo custo com características diferenciadas e alto valor agregado.

Palavras-Chave: Spirulina; biorremediação; aquicultura; tratamento de água residual.

\section{LIPID AND FATTY ACIDS COMPOSITION OF Spirulina sp. (LEB18) PRODUCED IN AQUACULTURE RESIDUAL WATER.}

\begin{abstract}
The objective was to evaluate the lipid and fatty acids composition of Spirulina sp. (LEB 18) grown in aquaculture wastewater. Cultures were performed in photobioreactors $(1 \mathrm{~L})$ with $100 \%$ residual aquaculture water supplemented with $\mathrm{T}-25$, T-50, T-75. The highest content of polyunsaturated fatty acids $(38,20 \%$ and $40,66 \%)$ and C18:3n6 (38,20\% and 33,50\%) were found in $25 \%$ and $50 \%$, respectively. Thus, the with $25 \%$ and $50 \%$ treatments represent an efficient, cheap and sustainable alternative for the aquaculture sector, reducing the impacts of effluent discharges, producing low cost biomass with different characteristics and high added value.
\end{abstract}

Keywords: Spirulina; bioremediation; aquaculture; wastewater treatment. 


\section{INTRODUÇÃO}

A aquicultura cresce a uma taxa constante de $4,5 \%$ ao ano. Em 2017, essa atividade industrial atingiu uma safra global total de 83,6 milhões de toneladas, representando $55 \%$ do total de peixes utilizados para consumo humano direto [1]. 0 Relatório do Banco Mundial estima que a aquicultura produza metade da oferta mundial de pescado até 2030 [2]. Segundo Wuang et al. [3], a aquicultura intensiva utiliza grandes quantidades de água doce limpa e gera fluxos de águas residuais ricas em nutrientes que podem causar a eutrofização das águas costeiras e impactar negativamente as comunidades biológicas a jusante.

A Spirulina sp. é fonte potencial de diversos compostos químicos. Dentre eles, os ácidos graxos que correspondem à maior fração lipídica, assim como representam os maiores teores de poliinsaturados (PUFA's) em $25 \%$ e $50 \%$ dos lipídios totais. Dessa forma, para Radmann et al. [4], os ácidos graxos quando extraídos podem ser utilizados como alimentos, fármacos ou transformados em bicombustíveis. Para isso, Morais et al. [5] consideram que para a produção de biodiesel é preferível que os ácidos graxos obtidos da biomassa sejam saturados, possuam alto número de cetano e que tenham menor capacidade de ocorrer oxidação do que os compostos insaturados.

Por outro lado, o crescimento da indústria de biomassa da Spirulina sp. e a aquicultura dependem diretamente da implementação de novos sistemas de cultivo destinados a reduzir custos e minimizar os impactos ambientais. Esta pesquisa visa aliar a produção de biomassa de Spirulina sp. através da reutilização e tratamento de águas residuais de aquicultura, determinando o melhor desempenho de produção através da composição de biomassa. Para atingir tal objetivo, o efluente aquícola foi suplementado com diferentes quantidades de nutrientes para favorecer o melhor desempenho de produção.

\section{METODOLOGIA}

\section{1. Água Residual da Aquicultura, Estirpe de Algas e Meio de Cultura}

O efluente aquícola foi gentilmente cedido pela Bahia Pesca S/A, localizada na Fazenda Joanes II, em Camaçari - Bahia (Nordeste do Brasil, 12ㄴ1'51"S e longitude 38ำ19'27"O). A cepa de Spirulina foi isolada da Lagoa Mangueira, no sul do Brasil (latitude 3232'05 "S e longitude 3331'57"O) e mantida em meio de Zarrouk conforme a metodologia de Costa et al. [6]. As cianobactérias Spirulina sp. (LEB 18) foi obtida da Coleção de Cultura do Laboratório de Engenharia Bioquímica da Universidade Federal do Rio Grande (FURG). 


\subsection{Preparação do Inoculo}

A água residual do cultivo de tilápia (Oreochomis niloticus) foi primeiramente suplementada com diferentes porcentagens do meio sintético de Zarrouk (ZM) $\mathrm{NaHCO}_{3} 16.8 \mathrm{~g} / \mathrm{L} ; \mathrm{NaNO}_{3} 2.5 \mathrm{~g} / \mathrm{L} ; \mathrm{NaCl} 1.0 \mathrm{~g} / \mathrm{L} ; \mathrm{K}_{2} \mathrm{HPO}_{4} 0.5 \mathrm{~g} / \mathrm{L} ; \mathrm{K}_{2} \mathrm{SO}_{4} 1.0 \mathrm{~g} / \mathrm{L}$; $\mathrm{MgSO}_{4} \cdot 7 \mathrm{H}_{2} \mathrm{O} 0.2 \mathrm{~g} / \mathrm{L} ; \mathrm{CaCl}_{2} 0.031 \mathrm{~g} / \mathrm{L} ; \mathrm{Na}_{2} E D T A 0.08 \mathrm{~g} / \mathrm{L} ; \mathrm{FeSO}_{4} \cdot 7 \mathrm{H}_{2} \mathrm{O} 0.01 \mathrm{~g} / \mathrm{L}$ [6]. Devido ao seu reconhecido equilíbrio nutricional, este meio de alga é o comumente utilizado para o cultivo da Spirulina sp. Os experimentos foram realizados apenas com a água residual da aquicultura (T-0) e com os respectivos tratamentos 25,50 e $75 \%$ dos nutrientes totais de Zarrouk (T-25, T-50 e T-75) seguindo proporções similares ao adotado por Kuo et al. [7] e Daneshvar et al. [8]. Após a suplementação, cada tratamento foi inoculado com Spirulina sp. (LEB 18) (no fim da fase exponencial). $\mathrm{O}$ crescimento foi monitorado por 7 dias, após o período a biomassa foi coletada para avaliar a produção, caracterização de biomassa (proteína, lipídios e carboidratos) e eficiência da remoção de nutrientes (utilizado para quantificar o efeito potencial do tratamento causado pelo crescimento de Spirulina sp. (LEB18)). O lipídeo extraído da biomassa foi caracterizado e a qualidade potencial do biodiesel a ser produzido foi estimada.

\subsection{Procedimento Experimental}

A biomassa da Spirulina sp. (LEB18) foi obtida a partir de um tanque de pista $(210 \mathrm{~L})$ em cultura contínua $(0,1$ biomassa $\mathrm{g} / \mathrm{L})$ mantido com o meio Zarrouk. Os diferentes ensaios foram preparados em Erlenmeyer com um volume de trabalho de $1 \mathrm{~L}$ por meio da utilização da água residual de aquicultura $(800 \mathrm{ml})$ inoculada com a Spirulina sp. (LEB18) com concentração de $0,3 \mathrm{~g} / \mathrm{L}$ de biomassa viva. Os experimentos seguiram a metodologia proposta por Kuo et al. [7] e Daneshvar et al. [8].

Os tratamentos experimentais (T-25, T-50 e T-75) foram preparados com 0 fornecimento de fotobiorreatores de $1 \mathrm{~L}$ (água de aquacultura/inóculo) com 25, 50 e $75 \%$ do total de nutrientes médios de Zarrouk individualmente. O T-0 corresponde ao efluente aquícola sem suplementação de nutrientes e o controle ao meio sintético Zarrouk. A incubação foi realizada durante 7 dias a $30^{\circ} \mathrm{C}$ com fotoperíodo de 12 horas claro/escuro. $\mathrm{O}$ ar foi fornecido a todas as culturas e lâmpadas tubulares fluorescentes com uma intensidade de luz de 41,6 $\mu$ mol fótons $\mathrm{m}^{-2} \mathrm{~s}^{-1}$ foram usadas como fonte de energia.

\subsection{Composição de Ácidos Graxos}

Os ácidos graxos foram transmetilados com trifluoreto de boro hexano e os ácidos graxos individuais das biomassas foram identificados por cromatografia gasosa. Os ésteres metílicos de ácidos graxos (FAME) foram separados e identificados em um cromatógrafo a gás (Clarus 680; Perkin Elmer ${ }^{\circledR}$ ) com uma 
coluna DB-FFAP (30 $\mathrm{m} \times 0.32 \mathrm{~mm} \times 0.25 \mathrm{~mm}$ ) e equipados com um detector de ionização de chama. Os parâmetros de análise incluíram temperaturas do injetor e detector de $250^{\circ} \mathrm{C}$ e $280^{\circ} \mathrm{C}$, respectivamente. Utilizou-se o programa de temperatura a seguir: $150^{\circ} \mathrm{C}$ por $16 \mathrm{~min}$, aumentando em $2^{\circ} \mathrm{C} / \mathrm{min}$ até $180^{\circ} \mathrm{C}$. Essa temperatura foi mantida por 25 min e em seguida foi feito um aumento de $5^{\circ} \mathrm{C} / \mathrm{min}$ até $210^{\circ} \mathrm{C}$, mantido por $25 \mathrm{~min}$. Foi utilizado hélio como gás de arraste a $1.0 \mathrm{~mL} \mathrm{~min}^{-1}$. Um fluxo de gás hidrogênio e de ar sintético foi fornecido em 30 e $300 \mathrm{~mL} \mathrm{~min}{ }^{-1}$, respectivamente. As injeções foram realizadas em duplicata para cada extração em um volume de $1 \mu \mathrm{L}$. Os FAME foram identificados comparando os tempos de retenção com uma mistura de padrões conhecidos (C4-C24, 18, 919-AMP; SigmaAldrich $\left.^{\circledR}\right)$. A quantificação dos ácidos graxos, expressa em $\mathrm{mg} \mathrm{g}^{-1}$ de lipídeos foi realizada pela adição do padrão interno tricosanoato de metila (C23:0; Sigma Aldrich $^{\circledR}$ ). As áreas dos picos foram determinadas através da metodologia de Nascimento et al. [9] utilizando o software de estação de tratamento de cromatografia para normalizar a porcentagem de áreas de ácidos graxos totais.

\section{RESULTADOS E DISCUSSÃO}

Os resultados apresentados na Tabela 1 mostram que os principais ácidos graxos são C16:0, C18:3n6 e C18:1n9-9. Segundo Malibari et al. [10], o ácido oleico (C18:1n-9) foi encontrado em percentagens elevadas em MUFAs sintetizadas por Chlorella sp., Dunaliella sp. e Tetraselmis sp. cultivada em águas residuais de fazendas de camarão. De acordo com Matos et al. [11] e baseado na literatura, C16 e C18 são dois ácidos graxos principais que são produzidos por processos enzimáticos e alongamento da cadeia de carbono e reações de desnaturação. Sendo assim, Gao et al. [12] observaram que as composições de ácidos graxos de Chlorella sp. o G-9, cultivado em águas residuais foi obtido com diferentes proporções de carbono orgânico total / nitrogênio total, consistindo principalmente de (C16:0), (C18:2) e (C18:3), que foi de 20,5-23,5\%, 25,4-29,4 \% e 11,7-19,8\%, respectivamente.

Tabela 1. Composição dos ácidos graxos da Spirulina sp. (LEB18) cultivada em 100\% da água residual da aquicultura suplementada com diferentes concentrações de Zarrouk

(Controle, T-25, T-50, T-25, T-0).

\begin{tabular}{|c|c|c|c|c|c|c|}
\hline \multirow{2}{*}{$\begin{array}{l}\text { Ácidos } \\
\text { Graxos }\end{array}$} & \multirow{2}{*}{$\begin{array}{l}\text { Nome } \\
\text { comum }\end{array}$} & \multirow[b]{2}{*}{ Controle } & \multirow[b]{2}{*}{ T-25 } & \multicolumn{3}{|c|}{ Tratamento } \\
\hline & & & & $\mathrm{T}-50$ & $\mathrm{~T}-75$ & $\mathrm{~T}-0$ \\
\hline $11: 0$ & Hendecanóico & $\begin{array}{c}1.65 \pm \\
0.08\end{array}$ & $\begin{array}{c}2.96 \pm \\
0.26\end{array}$ & $\begin{array}{c}8.05 \pm \\
0.12\end{array}$ & $\begin{array}{c}2.77 \pm \\
0.02\end{array}$ & $\begin{array}{c}3.76 \pm \\
0.08\end{array}$ \\
\hline $14: 0$ & Mirístico & $\begin{array}{c}0.84 \pm \\
0.02\end{array}$ & $\begin{array}{c}3.56 \pm \\
1.08\end{array}$ & $\begin{array}{c}4.48 \pm \\
0.89\end{array}$ & ND & ND \\
\hline $16: 0$ & Palmítico & $\begin{array}{c}31.43 \pm \\
4.03\end{array}$ & $\begin{array}{c}48.12 \pm \\
0.51\end{array}$ & $\begin{array}{c}44.79 \pm \\
0.51\end{array}$ & $\begin{array}{c}48.60 \pm \\
0.79\end{array}$ & $\begin{array}{c}49.65 \pm \\
0.46\end{array}$ \\
\hline 18:0 & Esteárico & ND & ND & $\begin{array}{c}2.08 \pm \\
0.20\end{array}$ & ND & ND \\
\hline $16: 1 n 7$ & Palmitoleico & $\begin{array}{c}2.18 \pm \\
0.97\end{array}$ & ND & ND & ND & ND \\
\hline $18: 1 n 9$ & Oleico & $10.58 \pm$ & $12.66 \pm$ & $9.31 \pm$ & 10.43 & $10.52 \pm$ \\
\hline
\end{tabular}




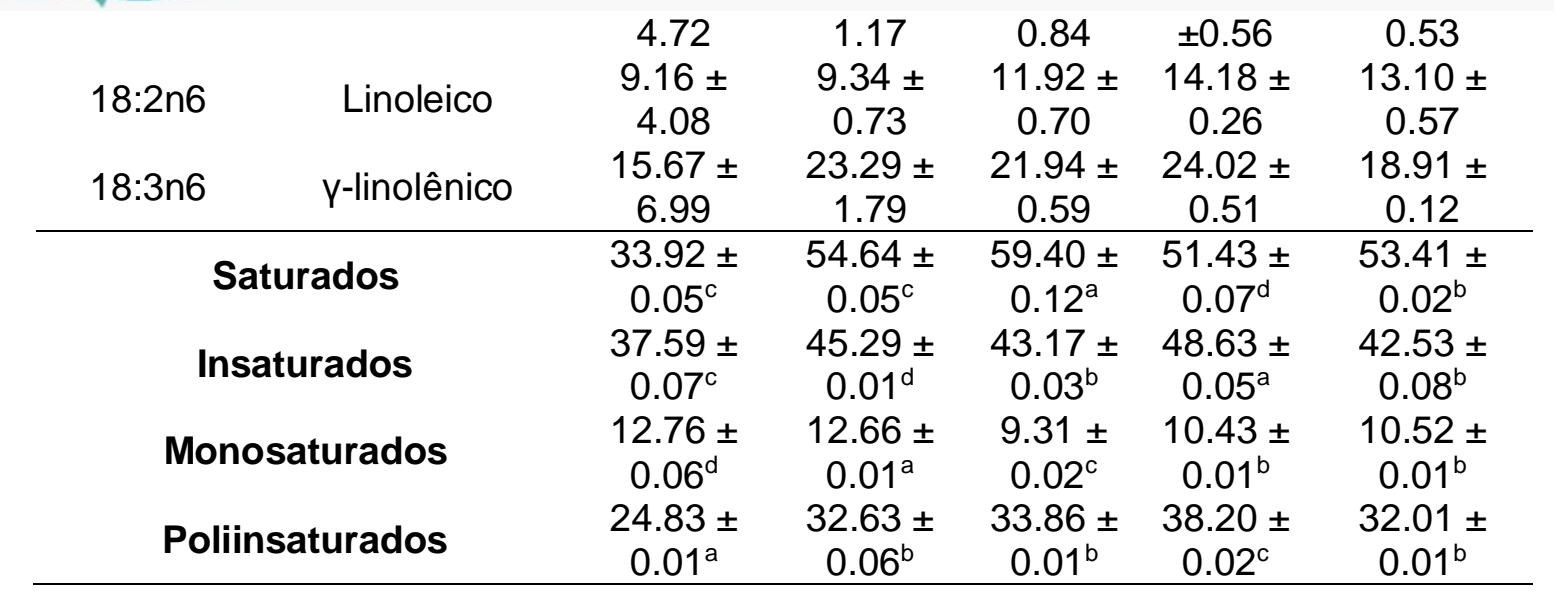

Média \pm desvio padrão; Letras equivalentes sobrepostas (a-d) na mesma linha indicam que não houve diferença significativa entre os valores no nível de confiança de $95 \%$. ND = Não identificado.

Conforme Kuo et al. [7], o efluente aquícola foi previamente reconhecido como meio adequado para o cultivo de Chlorella sp. com o objetivo de coleta do óleo. Os autores associaram boa qualidade do biodiesel obtido, à presença significativa de ácidos graxos C16:0, C18:0, C18:1 e C18:2. Entretanto, Nascimento et al. [9] inferem que essa combinação gera um biodiesel com atrasos de ignição curtos resultantes do alto nível de cetona. A Tabela 1 mostra que o T-25 e T-50 resultaram em altas concentrações de ácidos graxos saturados, denotando seu potencial para produzir um biodiesel de alta qualidade.

Os ácidos graxos saturados variaram entre 34 e $60 \%$ de todas as amostras, sendo assim Malibari et al. [10] evidenciou que os lipídios produzidos em efluentes de fazendas de camarão têm maior teor de ácidos graxos saturados, dentre eles os poliinsaturados e monoinsaturados variaram entre 24-38\% e $9-12 \%$, respectivamente. Ge et al. [13] observaram que os ácidos graxos insaturados, principalmente poli-insaturados, por possuírem ligações duplas podem sofrer degradação biológica e necessitar de uma posterior hidrogenação catalítica parcial, além de serem suscetíveis à oxidação durante o armazenamento. Ademais, para Freire et al. [14] e Zhang et al. [15] os ácidos graxos insaturados podem ser usados na alimentação de espécies marinhas fornecendo energia aos animais, formando componentes da membrana celular e substâncias altamente bioativas, regulando o metabolismo lipídico e apoiando a função imunológica.

Em estudo realizado por Santos et al. [16] abordou que a biomassa de Spirulina sp. produzida em escala piloto é rica em ácidos graxos poliinsaturados de valor comercial, como C18:3n6. No presente estudo, esse ácido graxo compreendeu $16 \%$ do total de lipídios produzidos no T-25. Em relação ao ácido gamalinolênico (C18:3n6), Perini et al. [17] relataram o papel importante deste ácido graxo na prevenção de doenças cardiovasculares e aterosclerose, doenças inflamatórias crônicas, inibição de vasoconstrição e agregação plaquetária, no crescimento fetal e desenvolvimento neural, ação anti-inflamatória e antitrombótica, ação sobre a prevenção do câncer e participação nas funções imunomoduladoras. Além disso, segundo Pokhrel et al. [18], o tratamento com T-25 também mostrou uma produção significativa de C16:0; que é essencial para o consumo humano e animal. Portanto, 
o uso de águas residuais da aquicultura suplementada com $25 \%$ de nutrientes de Zarrouk é uma estratégia adequada para afetar positivamente o teor de ácidos graxos de Spirulina sp.

\section{CONCLUSÃO}

O estudo demonstrou o desempenho de crescimento da Spirulina sp. (LEB18) em águas residuais de aquicultura suplementadas com quantidades distintas de nutrientes apresentou perfil de ácidos graxos com significativo valor nutricional humano e animal, juntamente com os constituintes certos para a produção de um biodiesel de alta qualidade. Assim, a Spirulina sp. (LEB18) cultivada em água residual do cultivo de Oreochomis niloticus suplementado com $25 \%$ e $50 \%$ de Zarrouk, representa uma alternativa sustentável para melhorar as condições ambientais da prática aquícola, reduzindo os impactos das descargas de águas residuais e obtendo biomassa com baixo custo, propriedades diferenciadas e de alto valor agregado.

\section{Agradecimentos}

Todos os autores agradecem o apoio da FAPESB - Fundação de Amparo à Pesquisa da Bahia no projeto CNPQ (400710 / 2014-5), MCTIC (Ministério da Ciência e Tecnologia da Informação e Comunicação) - Brasil e Bahia Pesca.

\section{REFERÊNCIAS}

${ }^{1}$ FOOD AND AGRICULTURE ORGANIZATION OF THE UNITED NATIONS. The State of World Fisheries and Aquaculture. Meeting The Sustainable Development Goals, Rome, 223 p., 2018.

${ }^{2}$ WORLD BANK REPORT. Fish to 2030: prospects for fisheries and aquaculture. The World Bank, Washington, DC, n. 83177, dez. 2013.

${ }^{3}$ WUANG, S. C. CHUA, P. Q. D. KHIN, M. C. LUO, Y. D. Use of Spirulina biomass produced from treatment of aquaculture wastewater as agricultural fertilizers. Algal Research, v. 15, p. 59-64, abr. 2016.

${ }^{4}$ RADMANN, E. M. COSTA, J. A. Conteúdo lipídico e composição de ácidos graxos de microalgas expostas aos gases $\mathrm{CO}_{2}, \mathrm{SO}_{2}$ e $\mathrm{NO}$. Química Nova, v. 31, n. 7, p. 1609-1612, set. 2018.

${ }^{5}$ MORAIS, M. G. COSTA, J. A. Perfil de ácidos graxos de microalgas cultivadas com dióxido de carbono. Ciência e Agrotecnologia, v. 32, n. 4, p. 1245-1251, jul/ago 2008. 
${ }^{6}$ COSTA, J. A. V. COLLA, L. M. FILHO, P. D. KABKE, K. et al. Modelling of Spirulina platensis growth in fresh water using response surface methodology. World Journal of Microbiology and Biotechnology, v. 18, p. 603-607, 2004.

${ }^{7} \mathrm{KUO}, \mathrm{CM}$. CHEN, TY. LIN, TH. KAO, CY. et al. Cultivation of Chlorella sp., GD using piggery wastewater for biomass and lipid production. Bioresource Technology, v. 194, p. 326-333, out. 2015.

${ }^{8}$ DANESHVAR, E. ANTIKAINEN, L. KOUTRA, E. KORNAROS, M. et al. Investigation on the feasibility of Chlorella vulgaris cultivation in a mixture of pulp and aquaculture effluents: Treatment of wastewater and lipid extraction. Bioresource Technology, v. 255, p. 104-110, jan. 2018.

${ }^{9}$ NASCIMENTO, I. A. MARQUES, S. TELES. I. CARVALHO, G. C. et al. Microalgae Versus Land Crops as Feedstock for Biodiesel: Productivity, Quality and Standard Compliance. BioEnergy Research, v. 7, p. 1002-1013, mar. 2014.

${ }^{10}$ MALIBARI, R. SAYEGH, F. ELAZZAZY, A. M. BAESHEN. M. N. et al. Reuse of shrimp farm wastewater as growth medium for marine microalgae isolated from Red Sea e Jeddah. Journal of Cleaner Production, v. 198, p. 160-169, jul. 2018.

${ }^{11}$ MATOS, Â. P. MOECKE. E. H. S. SANT'ANNA, E. S. The use of desalination concentrate as a potential substrate for microalgae cultivation in Brazil. Algal Research, v. 24, p. 505-508, jun. 2017.

${ }^{12} \mathrm{GAO}, \mathrm{F}$. YANG, HL. LI, C. PENG, YY. et al. Effect of organic carbon to nitrogen ratio in wastewater on growth, nutrient uptake and lipid accumulation of a mixotrophic microalgae Chlorella sp. Bioresource Technology, v. 282, p. 118-124, jun. 2019.

${ }^{13} \mathrm{GE}$, S. CHAMPAGNE, P. Nutrient removal, microalgal biomass growth, harvesting and lipid yield in response to centrate wastewater loadings. Water Research, v. 88, p. 604-612, jan. 2016.

${ }^{14} \mathrm{FREIRE}$, I. CORTINA, A. BARREIRO, P. LLAMAS, B. et al. Nannochloropsis limnetica: A freshwater microalga for marine aquaculture. Aquaculture Research, v. 459, p. 124-130, 2016.

15ZHANG, L. PEI, H. YANG, Z. WANG, X. et al. Microalgae nourished by mariculture wastewater aids aquaculture self-reliance with desirable biochemical composition. Bioresource Technology, v. 278, p. 205-213, abr. 2019.

${ }^{16}$ SANTOS, C. UEBEL, L. COSTA, S. MIRANDA, A. et al. Outdoor pilot-scale cultivation of Spirulina sp. LEB-18 in different geographic locations for evaluating its growth and chemical composition. Bioresource Technology, v. 256, p. 86-94, mai. 2018.

${ }^{17}$ PERINI, J. STEVANATO, F. SARGI, S. VISENTAINER, J. et al. Ácidos graxos poliinsaturados n-3 e n-6: metabolismo em mamíferos e resposta imune. Revista de Nutrição, v. 23, n. 6, nov./dez. 2010. ISSN 1415-5273.

${ }^{18}$ POKHREL, A. SONI, P. Performance analysis of different rice-based cropping systems in tropical region of Nepal. Journal of Environmental Management, v. 197, p. 70-79, jul. 2017. 